\title{
Evaluating the Energy Efficiency of Software Defined Based Cloud Radio Access Networks
}

\author{
Raad S. Alhumaima, Hamed S. Al-Raweshidy \\ WNCC, School of Engineering, Design and physical sciences, Brunel University London, Uxbridge, \\ Middx., UB8 3PH, UK \\ (Raad.Alhumaima, Hamed.Al-Raweshidy)@brunel.ac.uk
}

\begin{abstract}
Densifying the communications network and integrating innovative technologies leads to increased Power Consumption (PC), along with increased signalling and degraded scalability. The latter can be mitigated by using Software Defined Networks (SDN), while Cloud Radio Access Network (C-RAN) reduces the PC. Since evaluating and improving the PC is an important key success factor for the upcoming 5G generations, a reliable Power Model (PM) is required. This paper proposes a componentised, linear and parameterised PM, and explores the individual components relevant for PC analysis, particularly for Software Defined Cloud-Radio Access Network (SDC-RAN) architecture. The model quantifies the Energy Efficiency (EE) by capturing the PC of individual components, and measures the amount of PC in the network. Cooling and total PC of C-RAN and SDC-RAN for different parameters such as varying numbers of antennas and different system's bandwidth share has also been considered. The results show that SDC-RAN increases the total PC by about $20 \%$ compared to C-RAN. Additionally, the paper shows the results of modelling the participating Core Network's $(\mathrm{CN})$ control plane unit's PC along with establishing the accuracy of the components and the parameterised models.
\end{abstract}

\section{Introduction}

The relentless growing number of connected mobile devices, along with the abundance of new types of bandwidth-hungry applications, has meant high data rate demands and a huge amount of signalling within the Core Network (CN) [1]. According to Cisco, mobile data traffic is expected to intensify by about 11-fold between 2013 and 2018. Furthermore, mobile device connections will grow to about 10.5 billion by 2018 compared to 7.2 billion in 2013 [1], [2]. Additionally, Ericsson reportedly forecasts that in 2021, 150 billion devices will be 5G connected, up from 4.100 billion connections using LTE technology [3]. These rising numbers are alarming and should urge mobile operators to seek out innovative ideas, designs, protocols and advanced digital signal processing (DSP) techniques in order to effectively cope with this explosively high demand for data, while simultaneously providing scalable and faster connectivity [4].

In view of this demand, Cloud Radio Access Network (C-RAN) has been suggested by both mobile operators and equipment vendors to introduce cloud-computing in $5 \mathrm{G}$ cellular networks by pooling the Base Band processing Units (BBUs) in a shared and centralised data processing centre [5], known as a BBU pool.

In contrast to the legacy eNodeB, the main baseband physical procedures, cooperation and pro- 
cessing of the upper layers in C-RAN are executed in the BBU pool, whereas the simple Radio Frequency (RF) functions are tackled by the low Power Consumption (PC) Remote Radio Heads (RRHs) [6]. C-RAN therefore truncates the Capital Expenditures (CAPEX) and Operational Expenditures (OPEX) due to lower site leases, reduced maintenance cost and fewer site visits [7].

Other benefits of C-RAN include: (i) Using advanced DSP, coordination and cognitive radio techniques to process signals through any neighbouring BBU(s) and efficiently utilizing the available spectrum [8]; (ii) Managing traffic variations by exploiting fewer computing resources, and therefore, not utilising unwanted processors; (iii) Reducing cooling PC as well as the total PC.

Because densifying the network with large number of RRHs increases the signalling cost in the $\mathrm{CN}$ in relation to the Users' (UEs) handover set ups and authentications, techniques to improve the network scalability need to be extensively investigated. Recently, the research community has embraced SDN-LTE integration, which offers centralised administration for the underlying devices in the BBU pool. This will eventually give us scalable, easier to configure, more efficient and faster network design [9], [10].

Essentially, SDN architecture consists of three main components: Open Flow (O.F) switch, a controller and a channel (O.F protocol). The principle is to extract/decouple the control plane from/and data plane in the network's devices. Consequently, these two planes interact using O.F interfaces. As a result, the functions of control planes can be combined or centralised in a unified controller rather than in a set of distributed devices bound with a stringent control plane. The controller in turn sustains the software abstract and presents Application Programming Interfaces (APIs) to the network providers [11]. The O.F switch consists of flow tables, which are installed, modified and deleted by the controller using the O.F protocol to enable the switch evaluating the received traffic in terms of the packet's contents [12]. The flow tables comprise: i) flow entries that hold a group of match fields (such as Quality of Service (QoS) type, IP address, MAC address, packet priority, etc), and ii) actions, those executed by the switch to process the received packets. Once the switch receives a packet, it examines its flow tables to search for a match. Matching means that the incoming packets will be compared against the entry's fields to know if the packets are eligible to be handled or not. If the packet's content match the installed entry fields, the packets will be processed over an action correlated to that entry. These actions might include forwarding the packets to a specific port, dropping or flooding the packet to all ports [13]. The SDN layer is generally built on top of SDN controller, and should not obstruct or impede the legacy OSI network layers. Therefore, the signalling burden on core network elements such as Mobility Management Entity (MME), Serving Gateway (SGW) and Packets Gateway (PGW) can be relieved, resulting in the recovery of their upgrade cost.

However, bringing this idea to the C-RAN requires a comprehensive comparison for C-RAN with and without the use of SDN [10]:

1. Decentralising or separating the control and data plane by using SDN simplifies the design of the CN's entities (i.e. SGW, PGW and MME, etc.) by transferring part of the control plane's functions to the controller. This in turn decreases $\mathrm{CN}$ entities's overhead and therefore reduces their functional complexities.

2. Implementing SDN can benefit C-RAN more than Distributed RAN (D-RAN), as C-RAN exhibits unified infrastructure and management capabilities. Because of that, the signalling delay amongst the BBUs and the controller can be significantly reduced when connecting near located BBUs rather than distributed BBUs. However, when pooling the BBUs, new protocols, smart SON systems and programming based management systems will be neces- 
sary to ensure the full benefits of C-RAN, SDN offers a solution by dominating the BBU pool's administration.

3. In contrast to D-RAN, C-RAN deploys small coverage RRHs to bring the cell site closer to the UEs, which shortens the transmission distance. Equivalently, SDN while deploying O.F switches, it also brings the CN's contents closer to the UEs. The flow tables of the switches allow direct communication with the UEs, which means forwarding UEs' packets to their destination before approaching the BBU pool. This procedure further relaxes the latency bottleneck.

4. C-RAN is considered a green architecture compared to D-RAN. In addition, there is a demand to develop and launch new services, policies and applications to unleash the maximum potential of the network. This requires flexible and a programmable based paradigm such as SDN [14].

5. SDN is designed for wired and not for wireless networks. This consequently introduces complexity and causes inherent weakness to its effective deployment, since it requires an isolated and non-interfering wireless channel amongst the controller-switches and switchesRRHs. Eventually, power allocation and optimisation methods will be required amongst the mentioned parties.

While gaining the benefits of SDN, the conjectural increase in PC due to the addition of SDN devices to C-RAN can be neutralised by the following trends: (i) the legacy X-2 interface signalling cost amongst the BBUs is now partially or fully relieved, as the controller administrates the signalling amongst the BBUs, (ii) by means of Network Function Virtualization (NFV), which complements SDN to relocate the functions of network from dedicated devices to general servers, resulting in the potential provision of fewer hardware and computational devices that will reduce the PC, and (iii) replacing the currently deployed switches and routers with O.F switches only will cost the network the PC of the controller, not the O.F switches, therefore, the overall PC can be further reduced. Accordingly, based on the aforementioned demonstrations and the benefits of integrating SDN and C-RAN, it is required to assess the power cost and the overall price of such investment by comparing the PC of C-RAN and SDC-RAN. As a final introductory comment, PC likely means Power Consumption or Consumed.

\subsection{Related Work}

Several proposals have been published to describe either the $\mathrm{PC}$ of a certain component within the Base Station (BS), or the PC of all the components on the system level. In [15], the Radio Frequency (RF), subsystem PC model emulation is investigated, whilst the Power Amplifier (PA) on-off effect on the BS PC is presented in [16]. In [17] and [18], complex LTE BSs PMs are presented to estimate the PC of the State of the Art (SotA) BSs, the presented models sweep through the functional complexity of each subcomponent to evaluate the total PC. These models are found to be complex by the Energy Aware Radio and network TecHnologies (EARTH) project PMs in [19] and [20]. These are used to parameterise and simplify the BSs PM of [17] and [18]. The EARTH PM is used to draw and shape the SotA BSs PC when the PC of a single BS can be linearised, but it does not address the upcoming hybrid network's PC such as SDN based LTE system, Heterogeneous based C-RAN (H-CRAN) and the standalone C-RAN deployment. Finally, a simple experimental based PC of the SDN switch is modelled in [21]. 


\subsection{Main Contributions}

By introducing SDN to C-RAN, the former will surely escalate the overall network's PC as new devices, i.e., the SDN controller and O.F switches will be added to the network. Therefore, evaluating the $\mathrm{EE}$ of such architecture is essential to evaluate the power cost due to these additions. Failure to consider these factors has shaped the motivation for our investigations as follows:

1. Evaluating the components' or subsystems' PM to evaluate the PC within SDC-RAN. This work allows the network operators and vendors to measure the EE of each part prior to deployment.

2. Wrapping the functions' complexity of each component results in a simplified parameterised model, in which the complex representation of the network PC has been extensively reduced. Furthermore, the parametrised model can be used to evaluate the future power saving approaches in SDC-RAN, such as transmission power reduction, antenna deactivation and sleep modes techniques.

3. Providing a comparison of C-RAN and SDC-RAN cooling and total PC by considering the different network characteristics and parameters, such as the transmitted power, complexity of functions, optical budget, number of antennas and bandwidth sweep. This in turn shapes a long-term/large-scale PM for holistic EE analysis.

4. Modelling the CN's control plane units together with SDN's PC participants contribute to the analysis, which in turn gives additional realistic visualisation to the PC modelling.

5. The accuracy comparison of both components and parameterised models have been presented to show the degree in which the former can meet the latter.

The structure of this paper is as follows. In Section 2, the component's PM of SDC-RANs and its corresponding PC components are introduced. The total PC of the network is described in Section 3. Section 4 presents the parameterised PM. Section 5 provides selected simulation results. Finally, the conclusion is given in Section 6.

\section{Component's Power Model}

The component's PM can be defined by two main parts:

- The CN's PC, which consists of three parts: (i) the BBU pool (ii) control plane units and (iii) SDN units.

- RRHs, with MIMO consideration.

Both the $\mathrm{CN}$ and the RRH PMs have been divided into several sub components, each component contributes to the total PM. Fig. 1 shows the block diagram and the PC modules of the SDC-RAN.

\subsection{CN's Power Consumption $\left(P_{C N}\right)$}

This encompasses mainly the BBU pool, the control plane components (i.e. MME, SGW and PGW) and SDN components (SDN controller and SDN switch). The detailed CN's PC is described in the following subsections: 


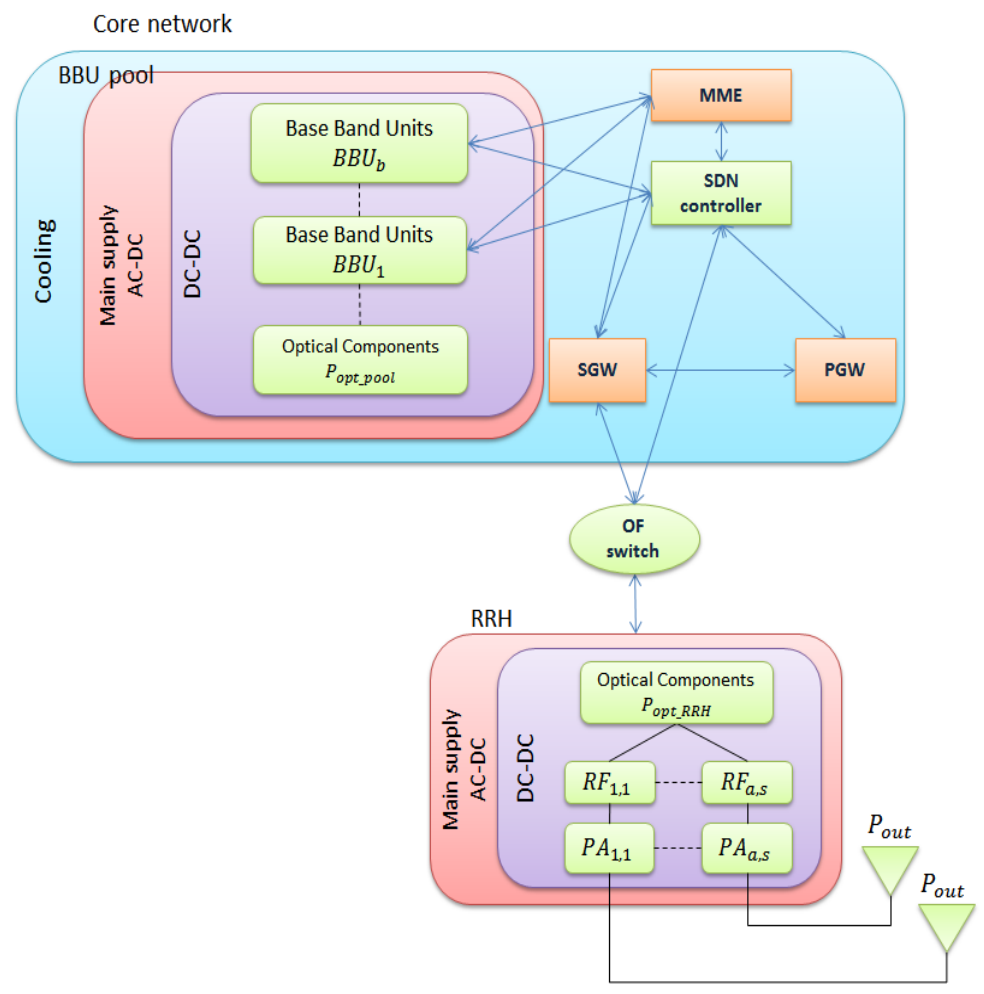

Fig. 1: Block diagram of SDC-RAN transceiver.

2.1.1. BBU power consumption $\left(P_{B B U}\right)$ : The $\mathrm{PC}$ for the $\mathrm{BBU}$ pool is considered to be the sum of the active BBUs in the BBU pool. The BBU is responsible for DSP and signal generation before passing it to the RF transceiver. The digital computation and processing of the BBU can be measured in Giga Operation per Second (GOPS) and translated into power figures. This can be achieved by multiplication of the GOPS with the technology scaling factor revealing the operations performed per second per Watt (W) [18]. A set of different BBUs functions ( $\left.I_{B B U}\right)$ such as the time and frequency domain processing, Forward Error Correction (FEC), Central Processing Units (CPU) and processing related to Common Public Radio Interface (CPRI) can be associated with GOPS [18]. The PM of BBU can be expressed as

$$
P_{B B U}=\sum_{i \in I_{B B U}} P_{i, B B U}^{r e f} A^{x_{i}^{A}} B^{x_{i}^{B}}
$$

$P_{i, B B U}^{r e f}$ in W, denotes the BBU's $i$-th function PC with respect to reference value (re f). A stands for the total number of Antenna/ RF transceiver. $x_{i}^{A}$ is the BBU scaling exponent of the number of RF chains. $B$ is the share of the bandwidth used, in Hz. Finally, $x_{i}^{B}$ is the scaling exponent of $B$.

2.1.2. Control plane PC $\left(P_{c l}\right)$ : Similarly to BBU mode of operation, MME, SGW and PGW, each is accountable for several functions within the network. For example, MME is responsible for roaming, authentication, authorisation, bearer management functions, UE's handover, etc. SGW and PGW in turn, are in control of many other functions. Eventually, these functions require digital computation and processing power which is then translated to GOPS. Although the type and number of functions in each subcomponent is different from the other, the way power is modelled is not 
affected. The control plane's PC can be expressed as the combination of the PC of the three main parts, i.e., MME $\left(P_{M M E}\right)$, SGW $\left(P_{S G W}\right)$ and PGW $\left(P_{P G W}\right)$, in W, each with its relative speed. By considering the set of functions associated with each unit. $P_{c l}$ in $\mathrm{W}$, then can be expressed as:

$$
\begin{aligned}
P_{c l} & =P_{M M E}+P_{S G W}+P_{P G W} \\
& =\sum_{q=1}^{Q} \sum_{m \in M_{M M E}}\left(E_{m, M M E}^{r e f} A^{y_{m}^{A}} B^{y_{m}^{B}}\right) v_{q}+\sum_{d=1}^{D} \sum_{s g \in S G_{S G W}}\left(E_{s g, S G W}^{r e f} A^{z_{s g}^{A}} B^{z_{s g}^{B}}\right) v_{d} \\
& +\sum_{g=1}^{G} \sum_{p g \in P G_{P G W}}\left(E_{p g, P G W}^{r e f} A^{j_{p g}^{A}} B^{j_{p g}^{B}}\right) v_{g}
\end{aligned}
$$

Where $M_{M M E}, S G_{S G W}$ and $P G_{P G W}$ designates the set of various functions in regards to MME, SGW and PGW respectively. $Q, D$ and $G$ symbolise the total number of MME, SGW and PGW respectively. $v_{q}, v_{d}$ and $v_{g}$, in packets per second (pps), denote the relative speed of each unit (the rate at which the packets are processed). $E_{m, M M E}^{r e f}, E_{s g, S G W}^{r e f}$ and $E_{p g, P g w}^{r e f}$, in Joule (J), denote the MME, SGW and PGW energy consumption of the $m$-th, $s g$-th and $p g$-th function corresponding to a reference value, respectively. $y_{m}^{A}, z_{s g}^{A}$ and $j_{p g}^{A}$ represent the scaling exponent of the number of RF chains that the MME, SGW and PGW serve, respectively. $y_{m}^{B}, z_{s g}^{B}$ and $j_{p g}^{B}$ stand for the scaling exponent of the bandwidth that MME, SGW and PGW exploit, respectively.

2.1.3. SDN power consumption $\left(P_{S D N}\right)$ : this can be further distributed into two main parts:

2.1.3.1. O.F switch power consumption $\left(P_{\text {switch }}\right)$ : the switch $\mathrm{PC}$ also can be divided into two sub components:

2.1.3.1.1. Port power consumption $\left(P_{\text {port }}\right): \quad$ each switch may contain several electronic chips (ports) used, each port with associated relative line speed.

$$
P_{\text {port }}=\sum_{o f=1}^{O F} \sum_{s w=1}^{S W} v_{s w}\left(E_{p r t, o f, s w}\right)
$$

$E_{p r t, o f, s w}$, in $\mathrm{J}$, indicates the energy consumed at full speed by $s w$-th port located in switch of. $v_{s w}$, in pps, is the line speed corresponding the $s w$-th port (the rate at which the packets are processed). $O F$ is the total number of O.F switches.

2.1.3.1.2. O.F Traffic power consumption $\left(P_{\text {flow }}\right)$ : The traffic or the flow represents the number of packets received by a particular O.F switch to be matched and accordingly actioned to the selected destination. $P_{\text {flow }}$, in $\mathrm{W}$, can be modelled as:

$$
P_{\text {flow }}=\sum_{o f}^{O F}\left(\sum_{f l}^{F L} R_{p k t}\left[\sum_{m t}^{M T} M C(f l, m t) E_{m t}+\sum_{d s}^{D S} N C(f l, d s) E_{d s}+\sum_{a k}^{A K} A C T(f l, a k) E_{a k}\right]\right)
$$


Where $F L$ symbolises the total number of flows received with an associated packet rate $\left(R_{p k t}\right)$, in pps. For each processed flow there is a possible number of matching, non-matching and actions $M C, N C$ and $A C T$ respectively. $M T, D S$ and $A K$ denote the total number of matched, nonmatched/discarded packets (the packets without matching in the corresponding flow table) and actions in each flow, respectively. $E_{m t}, E_{d s}$ and $E_{a k}$, in $\mathrm{J}$, refers to the energy required to take an action for the $m t$-th match, $d s$-th non-match packet and $a k$-th action, correspondingly. The total O.F switch PC $P_{\text {switch }}$, in W, then can be modelled:

$$
P_{\text {switch }}=P_{\text {port }}+P_{\text {flow }}
$$

2.1.3.2. SDN controller PC $\left(P_{S D N c t l}\right)$ : The controller PC can be expressed as the rates $R_{c t l, o f}^{O . F}$, $R_{c t l, q}^{M M E}, R_{c t l, d}^{S G W}$ and $R_{c t l, g}^{P G W}$, in pps, of outgoing O.F protocol control signalling from the $c t l$-th controller to the of -th O.F switch, $q$-th MME, $d$-th SGW and $g$-th PGW, correspondingly. Beside the corresponding energy $E_{c t l, o f}^{O . F}, E_{c t l, q}^{M M E}, E_{c t l, d}^{S G W}$ and $E_{c t l, g}^{P G W}$, in J/packet required to send the packet to the O.F, MME, SGW and PGW respectively. If $C T L$ is the total number of SDN controllers, the connection is bidirectional amongst the C-RAN control plane units and the controller, the PC can be modelled as:

$$
P_{S D N c t l}=\sum_{c t l=1}^{C T L}\left(\sum_{o f}^{O F} R_{c t l, o f}^{O . F}+2\left[\sum_{q=1}^{Q} R_{c t l, q}^{M M E} E_{c t l, q}^{M M E}+\sum_{d=1}^{D} R_{c t l, d}^{S G W} E_{c t l, d}^{S G W}+\sum_{g=1}^{G} R_{c t l, g}^{P G W} E_{c t l, g}^{P G W}\right]\right)
$$

Finally, the SDN's PC, in W, can be expressed as the aggregation of both the switch and the controller:

$$
P_{S D N}=P_{\text {switch }}+P_{S D N c t l}
$$

2.1.4. BBU pool DC-DC Conversion $\left(P_{D C, P}\right)$ : The various components of the BBU pool require proper DC voltages for operation therefore DC-DC converters are placed wherever needed. These DC-DC converters have efficiency less than 100\%, thus it can be expressed as a loss. It is mentioned in [20] and [19] that these losses incurred by the overhead (i.e. power conversions and active cooling) scale linearly with the $\mathrm{PC}$ of other components which require DC conversion, i.e. (BBUs, Optical devices, etc.). Therefore, the PC caused by the DC conversion $\left(P_{D C, P}\right)$, in W, of the BBU pool is given as:

$$
P_{D C, P}=\sum_{b=1}^{B} \sum_{r=1}^{R} l_{D C, P}\left(\eta_{D C, P}\right)\left[P_{B B U b}^{r}+P_{o p t, P_{b, r}}+P_{S D N}+P_{c l}\right]
$$

Where, $b \in\{1, \ldots, B\}$ stands for the number of active BBUs in the BBU pool; $r \in\{1, \ldots, R\}$ indicates the number of active RRHs; $P_{B B U b}{ }_{b}$, in W, is the PC of $b$-th BBU, which is connected to $r$-th RRH; $P_{o p t, P_{b, r}}$, in $\mathrm{W}$, is the PC by the optical device in the BBU pool which connects the $b$-th $\mathrm{BBU}$ to $r$-th RRH; $l_{D C, P}$ is the loss caused by DC-DC conversion as a function of DC conversion efficiency $\left(\eta_{D C, P}\right)$.

The loss function can be modelled as a decay function with an exponential decay constant $(x)$. This constant is subjected to the manufacturer design quality and eventually affects the loss 
function value of each component. More details can be found in Section 5, where a possible comparison of the ways to meet the practical considerations of the participating devices is given. However, the losses can be expressed by:

$$
l(\eta)=l_{o} e^{-\eta x}
$$

$l_{o}$ is the initial value of the losses at the time $(\mathrm{t}=0)$ and $\eta$ is the efficiency of the device. The efficiency can be modelled as the ratio of maximum DC conversion output power $\left(P_{D C, P, \text { out } \max }\right)$ to actual PC by the other DC power components.

$$
\eta_{D C, P}=\frac{P_{D C, P, \text { out }, \max }}{\sum_{b=1}^{B} \sum_{r=1}^{R} P_{B B U b}^{r}+P_{o p t, P b, r}+P_{S D N}+P_{c l}}
$$

2.1.5. Mains supply (MS), AC-DC Conversion PC $\left(P_{M S, P}\right)$ : The power form of the main supply grid has to be converted from AC to DC. This can be realized by the MS conversion unit. The PC of this unit, in $\mathrm{W}$, is generally modelled the same way as the DC-DC power conversion, and it is given as:

$$
P_{M S, P}=P_{D C, P}+\sum_{b=1}^{B} \sum_{r=1}^{R} l_{M S, P}\left(\eta_{M S, P}\right)\left[P_{B B U b}^{r}+P_{o p t, P_{b, r}}+P_{S D N}+P_{c l}\right]
$$

$l_{M S, P}$ represents the measured losses of the MS power conversion as a function of the MS conversion efficiency $\left(\eta_{M S, P}\right)$. The MS's efficiency is the ratio of the maximum AC converter output power $\left(P_{M S, P, \text { out }, \text { max }}\right)$, in $\mathrm{W}$, to the actual PC by the other components in the BBU pool:

$$
\eta_{M S, P}=\frac{P_{D C, P, o u t, \max }}{P_{D C, P}+\sum_{b=1}^{B} \sum_{r=1}^{R} P_{B B U b}^{r}+P_{o p t, P_{b, r}}+P_{S D N}+P_{c l}}
$$

2.1.6. Cooling: Cooling is responsible for a great energy waste. The cooling unit is responsible for cooling the entire components in the $\mathrm{CN}$. However, the PC is modelled to be proportional to the consumption of all other components in the BBU pool. If $l_{\text {cool }}$ is the cooling loss, the cooling PC $\left(P_{\text {cool }}\right)$, in $\mathrm{W}$, can be modelled as:

$$
P_{\text {cool }}=l_{\text {cool }}\left(P_{M S, P}+P_{D C, P}+\sum_{b=1}^{B} \sum_{r=1}^{R} P_{B B U b}^{r}+P_{o p t, P_{b, r}}+P_{S D N}+P_{c l}\right)
$$

\subsection{Remote Radio Head (RRH)}

The RRH consists of components with a much lower operational complexity than BBU pool. It is equipped with an RF and PA that scale linearly with the number of antennas, the RRH also consists of necessary voltage suppliers. However, RRHs PM can be broken down to the following components:

2.2.1. Antenna $(A)$ : The number of antennas used in the system affects the $P M$ as each antenna requires $\mathrm{RF}$ and $\mathrm{PA}$ to provide the necessary required signal operations and amplification. 
2.2.2. RF transceiver's $P C\left(P_{R F}\right)$ : The RF unit is responsible for several functions related to intermediate frequency and baseband interface such as a) Modulation/Demodulation of the signals, Voltage Controlled Oscillators (VCO) and Mixers, Digital to analogue (DAC) and analogue to digital (ADC) converters and Low Noise Amplifiers (LNAs), gain amplifiers and clocks.

The RF's PM $\left(P_{R F}\right)$, in W, can be expressed the same way as the BBU's PC:

$$
P_{R F}=\sum_{i \in I_{R F}} P_{i, R F}^{r e f} A^{x_{i}^{A}} B^{x_{i}^{B}}
$$

$P_{i, R F}^{r e f}$, in W, denotes the RF's $i$-th function's PC with respect to reference value (ref).

2.2.3. Power amplifier $\left(P_{P A}\right)$ : The $\mathrm{PA}$ is a prime element of consideration in the $\mathrm{PM}$, as it consumes most of the power within the network, especially in the RRH. The PA amplifies the electrical signal received from the $\mathrm{O} / \mathrm{E}$ convertor before its transmission to the air interface and vice versa. In rare cases, its efficiency can reach up to 54\% when transmitting high powers. This low efficiency comes as a results of the strong fluctuation in the powers of the OFDM signals [17]. However, modelling the PC of a PA requires the following parameters:

- Output transmitted power $\left(P_{\text {out }}\right)$, in $\mathrm{W}$, of the antenna.

- Output power of the PA $\left(P_{T X}\right)$, in W.

- The share of bandwidth $(B)$, in $\mathrm{Hz}$, that the antenna uses, i.e., the actual number of the Physical Resource Blocks $\left(N_{P R B}\right)$ that occupies a certain bandwidth for transmission; $P_{T X}=$ $P_{\text {out }} B$.

The PA's PC is also affected by its efficiency $\left(\eta_{P A}\right)$, which is a function of $P_{T X}$. Therefore the SotA PA's PC, in W, can be modelled as:

$$
P_{P A}=\frac{P_{T X}}{\eta_{P A}\left(P_{T X}\right)}
$$

2.2.4. RRH's Power Conversion: AC-DC and DC-DC voltage converters are required to provide the necessary voltage supplies to the RRH's components. The PC of the RRH's DC conversion $\left(P_{D C, R}\right)$, in $\mathrm{W}$, is modelled with consideration of conversion losses $\left(l_{D C, R}\right)$ as a function of the conversion efficiency $\left(\eta_{D C, R}\right)$, along with the power requirement of all other components within the RRH:

$$
P_{D C, R}=l_{D C, R}\left(\eta_{D C, R}\right)\left[P_{o p t, R}+\sum_{b=1}^{B} \sum_{r=1}^{R} \sum_{a=1}^{A}\left(P_{P A}+P_{R F a}^{r, b}\right)\right]
$$

$P_{R F a}^{r, b}, P_{P A a}^{r, b}$ in W, denote the PC of the $a$-th RF and PA respectively, within $r$-th RRH, which is connected to $b$-th BBU. $P_{o p t, R}$, in W, is the PC of the optical device inside the RRH. $\eta_{D C, R}$ is RRH's conversion efficiency, which is the ratio of the maximum output power of the DC converter $\left(P_{D C, R, \text { out }, \text { max }}\right)$, in $\mathrm{W}$, to the actual PC by other components.

$$
\eta_{D C, R}=\frac{P_{D C, R, \text { out }, \max }}{P_{o p t, R}+\sum_{a=1}^{A}\left(P_{R F}+P_{P A}\right)_{a}}
$$


The MS converter of the RRH can be modelled in the same fashion as the BBU pool's MS, therefore its PM, in $\mathrm{W}$, is configured as

$$
P_{M S, R}=l_{M S, R}\left(\eta_{M S, R}\right)\left[P_{D C, R}+P_{o p t, R}+\sum_{b=1}^{B} \sum_{r=1}^{R} \sum_{a=1}^{A}\left(P_{R F}+P_{P A}\right)_{a}^{r, b}\right]
$$

$l_{M S, R}$ symbolises the MS losses as a function of the conversion efficiency $\left(\eta_{M S, R}\right)$, which is modelled as:

$$
\eta_{M S, R}=\frac{P_{M S, R, o u t, \max }}{P_{D C, R}+P_{o p t, R}+\sum_{a=1}^{A}\left(P_{R F}+P_{P A}\right)_{a}}
$$

$P_{M S, R, \text { out }, \text { max }}$, in W, signifies the maximum output power of the RRH's MS converter.

2.2.5. RRH's cooling: Nokia Siemens Networks (NSN) states that BSs with total PC of $500 \mathrm{~W}$ or less (except the output power of the BS $\left(P_{\text {out }}\right)$ ) do not require a cooling system [16]. This is applicable for the RRH, which is composed of PA, RF and optical components. These components consume power less than $500 \mathrm{~W}$. Therefore, the only overhead considered for RRH is the power supplies [20].

\subsection{Optical transceiver's PC}

The optical transceivers are responsible for the electrical and optical conversions and vice versa. Moreover, the typical PC of commercial point-to-point (PtP) and point-to-multipoint (PtMP) optical transceivers are respectively $1 \mathrm{~W}$ and $1.5 \mathrm{~W}$ [22]. The proposed model uses PtP transceivers, as this type does not have a passive optical power splitter and offers a relaxed link budget. In other words, PtP link loss is governed by only the distance and the operating wavelength. This is in contrast to the PtMP, in which a constrained link budget of about (20-35) dB is required due to the wavelength sharing nature of the fiber, whereas the link loss of a PtP is as low as $6 \mathrm{~dB}$ within 20 $\mathrm{km}$ of network reach.

\section{Total power consumption $\left(P_{S D C-R A N}\right)$}

The total PC of the SDC-RAN network $\left(P_{S D C-R A N}\right)$, in W, is therefore the sum of the CN's PC $\left(P_{C N}\right)$ and RRHs' PC $\left(P_{R R H}\right)$, it is formulated as the following:

$$
P_{S D C-R A N}=P_{C N}+P_{R R H}
$$

$P_{C N}$, in $\mathrm{W}$, is calculated by aggregating the $\mathrm{PC}$ of the corresponding components:

$$
P_{C N}=P_{c o o l}+P_{M S, P}+P_{D C, P}+P_{S D N}+P_{c l}+\sum_{b=1}^{B} \sum_{r=1}^{R} \sum_{a=1}^{A} P_{B B U b, r, a}+P_{o p t, P_{b, r}}
$$


$P_{B B U b, r, a}$, in $\mathrm{W}$, denotes the PC of $b$-th BBU attached to $r$-th RRH mounting $a$-th antenna. RRHs can be served by any active BBU within the BBU pool. This means that BBUs-RRHs mapping can be dynamic depending on the traffic conditions. This service diversity is considered in the model. The total PC of the RRHs is formulated as:

$$
P_{R R H}=P_{M S, R}+P_{D C, R}+\sum_{b=1}^{B} \sum_{r=1}^{R} \sum_{a=1}^{A}\left(P_{P A}+P_{R F}\right)_{r, a}^{b}+P_{o p t, R_{r}}
$$

$P_{P A_{r, a}}^{b}, P_{R F_{r, a}}^{b}$ in W, symbolise the PC of the PA and RF respectively; of $a$-th antenna served by $r$-th RRH that is attached to $b$-th BBU.

\section{Parameterised and Linear Power Model}

The PC of the proposed SDC-RAN can be expressed by a load-independent share $\left(P_{\text {static }}\right)$ and an added load-dependent dynamic share that upsurges linearly with the power slope $(\Delta)$, as shown in Fig. 2. The maximum supply power $\left(P_{\text {supply }}\right)$ depends on the maximum transmitted power $\left(P_{\text {out }}\right)$ of the RRH, the share of bandwidth used $(B)$ and the number of radio chains $(A)$. The Maximum supply PC $\left(P_{1}\right)$ is reached when transmitting at maximum power $\left(P_{\text {out }}\right)$.

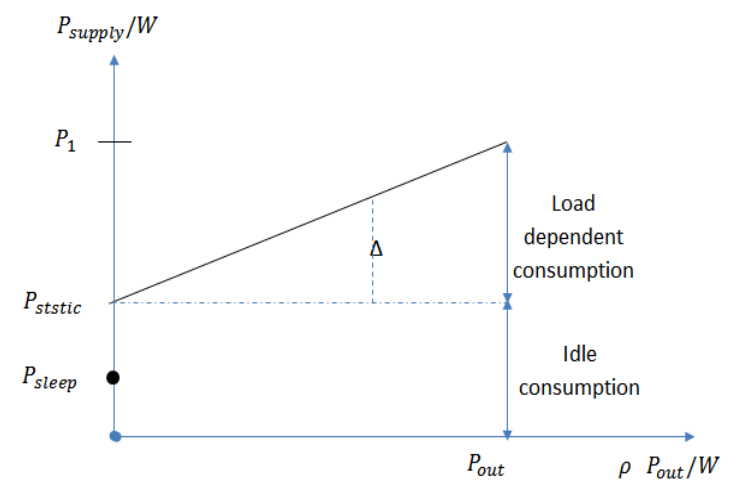

Fig. 2: BS Load-dependent PM.

Furthermore, when the RRH is not transmitting, it may enter into sleep mode with lower PC $\left(P_{\text {sleep }}\right)$. The total power supply is then formulated, in $\mathrm{W}$ :

$$
P_{\text {supply }}(\rho)= \begin{cases}(R)\left(P_{1}+\Delta P_{\text {out }}(\chi-1)\right) & \text { if } 0<\rho \leq 1 \\ (R) P_{\text {sleep }}, & \text { if } \rho=0\end{cases}
$$

$P_{1}=P_{\text {static }}+\Delta P_{\text {out }} ; \rho$ is the scaling parameter of the load share. If $\rho=1$, the system is fully loaded, e.g. transmitting at full bandwidth and power, whereas the system is idle when $\rho=0$. In order to briefly understand how different parameters contribute towards this model, the maximum supply PC $\left(P_{1}\right)$ is parameterised here.

Noticing that only few parameters are consistently varied within this model and most of the parameters remain static, the following analysis and approximations are made in relevance to the SotA BS's PM: 
- The BBU and RFs' PC, both scale linearly with the number of antennas $(A)$ and the bandwidth $(B)$, i.e., $P_{B B U}=A \frac{B}{10 M H z}\left(P_{B B U}^{p m}\right)$ and $P_{R F}=A \frac{B}{10 M H z}\left(P_{R F}^{p m}\right) . P_{B B U}^{p m}, P_{R F}^{p m}$ denote the $\mathrm{PC}$ by the $\mathrm{BBU}$ and $\mathrm{RF}$ in the parameterised model $(\mathrm{pm})$, respectively.

- The control plane components' PC i.e. (MME, SGW and PGW) scale linearly with the number of antenna and the bandwidth i.e. $P_{M M E}=A \frac{B}{10 M H z}\left(P_{M M E}^{p m}\right), P_{S G W}=A \frac{B}{10 M H z}\left(P_{S G W}^{p m}\right)$ and $P_{P G W}=A \frac{B}{10 M H z}\left(P_{P G W}^{p m}\right)$. The similar holds true for the O.F switch and the SDN controller PC, i.e. $P_{\text {switch }}=A \frac{B}{10 M H z}\left(P_{\text {switch }}^{p m}\right)$ and $P_{S D N c t r l}=A \frac{B}{10 M H z}\left(P_{S D N c t r l}^{p m}\right)$.

- The PC of PA depends on the maximum power transmitted per antenna $\left(P_{\text {out }} / A\right)$ and its efficiency $\left(\eta_{P A}\right)$. The feeder loss between PA and the antenna can be ignored for the RRH as the PA is placed close to the antenna. The PA's PC can be represented as $P_{P A}=\frac{P_{\text {out }}}{A \eta_{P A}}$.

- DC-DC, AC-DC conversions as well as cooling's PC, scale linearly with the other components PC and are approximated by the loss factors $\sigma_{D C, P}, \sigma_{M S, P}, \sigma_{c o o l}, \sigma_{D C, R}$ and $\sigma_{M S, R}$ for BBU pool DC, BBU pool MS, cooling, RRH's DC, and RRH's MS loss factors respectively.

- The optical transceivers' PC $P_{o p t, P}$ and $P_{o p t, R}$, scale linearly with the number of BBUs and RRHs in the network.

- The losses incurred by the cable's connections amongst the entities within the $\mathrm{CN}$ or the backhaul can be expressed by the loss factor $\left(\sigma_{\text {backhaul }}\right)$, moreover, it scales linearly with the numbers of BBUs.

- The losses incurred by the fronthaul optical fiber are estimated by the loss factor $\left(\sigma_{\text {fronthaul }}\right)$. Theses losses scale linearly with the numbers of RRHs and can be adapted to meet the fiber lengths and number of connectors and splices used.

The maximum PC $\left(P_{1}\right)$, in $\mathrm{W}$, can be formulated by aggregation of the $\mathrm{PC}$ of the $\mathrm{CN}$, which constitute of (BBUs, control plane, O.F switch and SDN controller) serving a single RRH:

$$
\begin{aligned}
P_{1} & =P_{C N}^{p m}+P_{R R H}^{p m} \\
& =\frac{A \frac{B}{10 M H z}\left(P_{B B U}^{p m}+P_{M M E}^{p m}+P_{S G W}^{p m}+P_{P G W}^{p m}+P_{\text {switch }}^{p m}+P_{S D N c t r l}^{p m}\right)+P_{o p t, P}}{\left(1-\sigma_{D C, P}\right)\left(1-\sigma_{M S, P}\right)\left(1-\sigma_{\text {cool }}\right)\left(1-\sigma_{\text {backhaul }}\right)} \\
& +\frac{A \frac{B}{10 M H z}\left(P_{R F}^{p m}\right)+\frac{P_{\text {max }}}{A \eta_{P A}}+P_{\text {opt }, R}}{\left(1-\sigma_{D C, R}\right)\left(1-\sigma_{M S, R}\right)\left(1-\sigma_{\text {fronthaul }}\right)}
\end{aligned}
$$

Then the total number of $R$ RRHs and $U$ BBUs is considered to obtain the total PC of the network $\left(P_{\text {supply }}\right)$, in W:

$$
P_{\text {supply }}=U \cdot P_{C N}^{p m}+R \cdot P_{R R H}^{p m}
$$




\section{Results and Discussion}

The proposed model is used to effectively analyse and identify the PC in terms of bandwidth, varying antenna numbers, and varying number of BBUs, RRHs and SDN devices. The parameters of the study were selected according to [20] and [21] when possible. The resulting parameters are provided in Table I, which summarises the SotA PC measurements based of the RRH and SDN devices.

Table 1 Parameters

\begin{tabular}{|c|c|c|}
\hline \multicolumn{3}{|l|}{ Breakdown } \\
\hline Component & Unit & Value \\
\hline$P_{\max }$ & $\mathrm{W}$ & 40 \\
\hline$P_{P A}$ & $\mathrm{~W}$ & 40 \\
\hline$P_{T X}$ & $\mathrm{~W}$ & 6.8 \\
\hline$P_{R X}$ & $\mathrm{~W}$ & 6.1 \\
\hline$P_{R F}^{p m}$ & $\mathrm{~W}$ & 12.9 \\
\hline$P_{B B U}^{p m}$ & $\mathrm{~W}$ & 29.4 \\
\hline$P_{M M E}^{p m}$ & $\mathrm{~W}$ & 65 \\
\hline$P_{S G W}^{p m}$ & $\mathrm{~W}$ & 65 \\
\hline$P_{P G W}^{p m}$ & $\mathrm{~W}$ & 68 \\
\hline$P_{\text {switch }}^{\text {pm }}$ & $\mathrm{W}$ & 58 \\
\hline$P_{S D N c t r l}^{p m}$ & W & 20 \\
\hline$P_{o p t, P}$ & W & 1 \\
\hline$P_{o p t, R}$ & $\mathrm{~W}$ & 1 \\
\hline$P_{1}$ & $\mathrm{~W}$ & 340 \\
\hline$P_{\text {static }, \text { sleep }}$ & $\mathrm{W}$ & 148 \\
\hline$\eta_{P A}$ & - & 0.31 \\
\hline$\sigma_{D C}$ & - & 0.075 \\
\hline$\sigma_{M S}$ & - & 0.09 \\
\hline$\sigma_{\text {backhaul }}$ & - & 0.085 \\
\hline$\sigma_{\text {fronthaul }}$ & - & 0.085 \\
\hline$\sigma_{\text {cool }}$ & - & 0.1 \\
\hline$\Delta$ & - & 2.8 \\
\hline$A$ & - & $1,2,4$ \\
\hline
\end{tabular}

The projected model is verified for one, two, and four antennas. $R$ is up to 60, while $U$ equals 20. Nevertheless, these numbers can be adjusted according to the network operator's architectural or configuration demands; this allows us to observe the variations in the individual parameters. The allocated bandwidth is $10 \mathrm{MHz}$; if increased, the PC is expected to increase as well. Fig. 3 shows the cooling PC of C-RAN and SDC-RAN of one, two and four antenna configurations. Due to the fact that the PA and RF components are no longer contributing to the cooling $\mathrm{PC}$ in the $\mathrm{BBU}$ pool, C-RAN reduces the cooling PC compared to the SotA BSs.

Fig. 4 shows the total network PC. By using the percentage change rule, i.e., $((V 1-V 2) /|V 1|) *$ $100 \%$ to compare the values in Fig.4, it was found that the total PC increasing percentage by adding SDN to the C-RAN architecture is about $20 \%$, for all antenna configurations. However, this value can be endangered to constraints such as operators' equipment PC and quality.

Fig.5 indicates the total PC as a function of the system's bandwidth share with varying numbers of antennas.

The accuracy of the simplified parameterised model and the components model can be evaluated and compared using the loss function (9). The main key comparison can be done by acknowledg- 


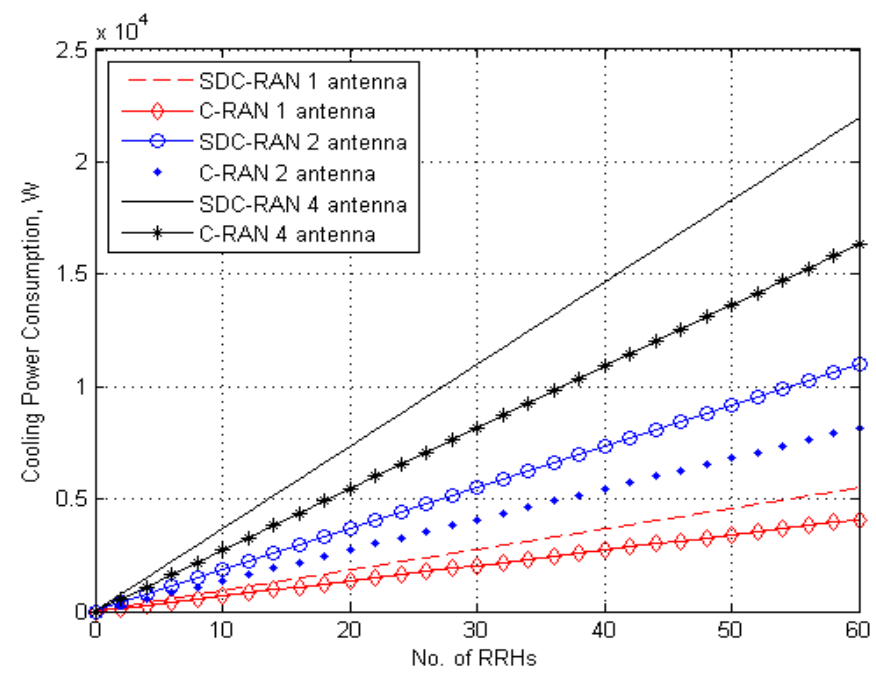

Fig. 3: Comparison the cooling PC of C-RAN and SDC-RAN, with one, two and four antennas configurations.

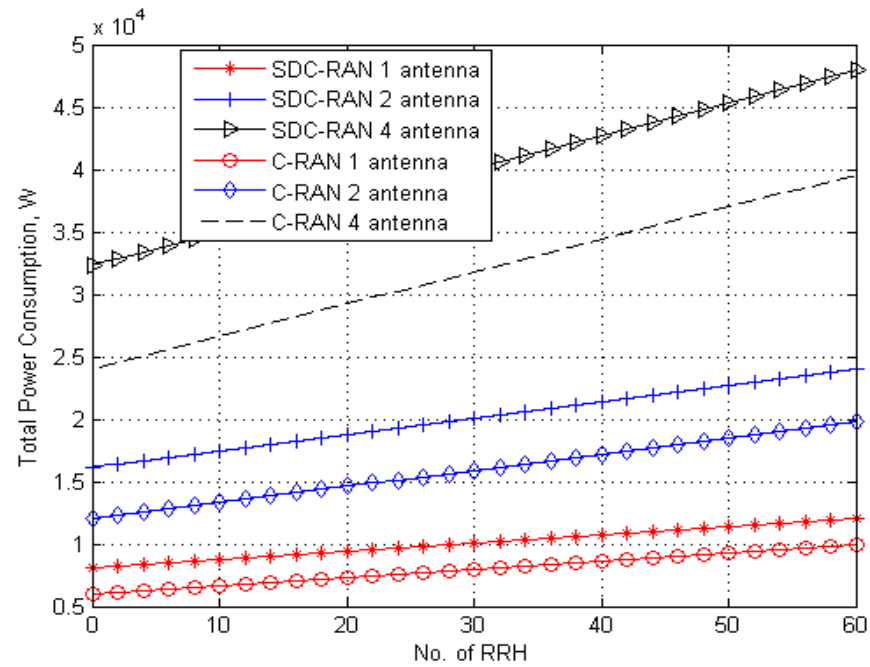

Fig. 4: Comparison of C-RAN and SDC-RAN total PC, 20 BBUs are used to serve 60 RRHs, with one, two, and four antennas configurations.

ing the initial losses $l_{o}$ of each subcomponent and the corresponding exponential decay constant $(x)$; the latter have been subjected to the manufacturer design. However, to compare both the components and the parameterised models in terms of accuracy, some assumptions have been made to the component PM:

1. The efficiency $\eta$ is $90 \%$ for all the sub components (i.e. AC, DC converters), and the initial loss $l_{o}$ is 0.009 .

2. (9) was used to evaluate the losses values which are required in (8), (11), (16), and (18).

3. The total component's PM were calculated using (20). 


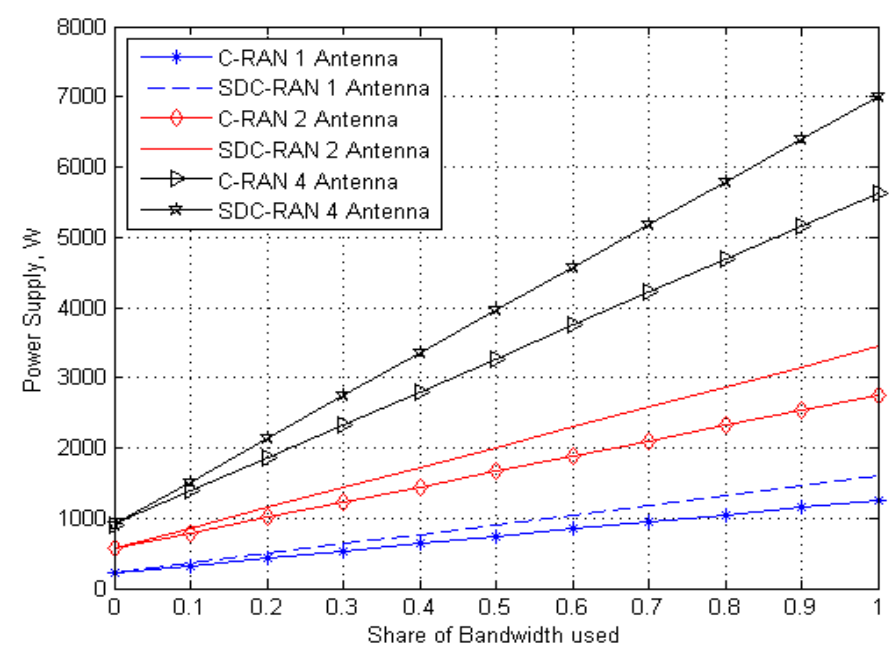

Fig. 5: Comparison of C-RAN and SDC-RAN total PC as a function of the used bandwidth (10 $\mathrm{MHz}$ ).

4. It was compared with the parameterised model of (24).

Based on the subcomponents' data sheet and by using different $x$ values, the results show as accurate parameterised as components PM. Fig (6) shows the total PC of SDC-RAN with different $x$ values and different number of RRHs.

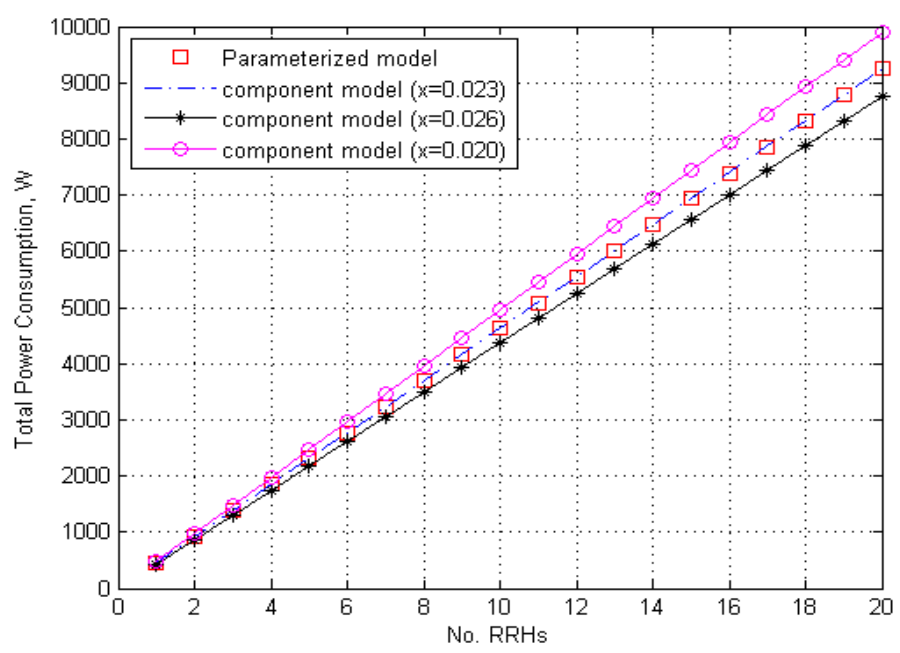

Fig. 6: Accuracy comparison of the total PC of the components and parameterised PMs with different values of $(x)$, using only one antenna configuration.

\section{Conclusion}

The components in conjunction with a parameterised PM are presented within this paper to demonstrate PC calculation of the extended network architecture SDC-RAN, according to operational 
parameters and the varying vendor configurations. The model is considered to visualise the PC cost, along with a comparison C-RAN and SDC-RAN models' performance regarding cooling and total PC, with varying parameters such as antenna and bandwidth sweep.

Intuitively, C-RAN model has less PC than SDC-RAN. However, the benefits demonstrated and the characteristics gained by the latter justifies the power cost, especially, when SDN units participate and cooperate the functional computations and the signalling process of the legacy control units to take over the administration and unleash the network potentials. Furthermore, SDN is advocated because of the capability and the flexibility within its architecture to introduce new services to the network and integrate with the new power reduction methods, such as NFV. However, the amount of power cost due to SDN deployment cannot be realised unless a reliable PM is introduced. This research resolves this ambiguity. Finally, the results show that the parameterised SDC-RAN is as accurate as the component's PM subject to equipment design quality.

\section{References}

[1] Zhou, T., Huang, Y., Fan, L., Yang, L.: 'Load-aware user association with quality of service support in heterogeneous cellular networks', Commun. IET., 2015, 9, (4), pp. 494-500.

[2] Yang, S., Hanzo, L.: 'Fifty Years of MIMO Detection: The Road to Large-Scale MIMOs', Commun. Surv. Tutorials, IEEE, 2015, 17, (4), pp. 1941-1988.

[3] Ericsson mobility report., available online: http://www.ericsson.com/res/docs/2015/mobilityreport/ericsson-mobility-report (Ericsson, Nov, 2015).

[4] Sciancalepore, V., Mancuso, V., Banchs, A.: 'BASICS: Scheduling base stations to mitigate interferences in cellular networks'. in World of Wireless, Mobile and Multimedia Networks (WoWMoM), IEEE 14th International Symposium and Workshops, 2013, pp. 1-9.

[5] Xiong, N., Han, W., Vandenberg, A.: 'Green cloud computing schemes based on networks: a survey', Commun. IET., 2012, 6, (18), pp. 3294-3300.

[6] Sundaresan, K., Arslan, M.Y., Singh, S., Rangarajan, S., Krishnamurthy, S. V: 'FluidNet: A Flexible Cloud-based Radio Access Network for Small Cells'. Proc. of the 19th Annual International Conference on Mobile Computing and Networking (ACM), 2013, pp. 99-110

[7] Peng, M., Zhang, K., Jiang, J., Wang, J., Wang, W.: 'Energy-Efficient Resource Assignment and Power Allocation in Heterogeneous Cloud Radio Access Networks', Veh. Technol. IEEE Trans., 2014, 64, (11), pp. 5275-5287.

[8] Ghamari Adian, M., Aghaeinia, H., Norouzi, Y.: 'Spectrum sharing and power allocation in multi-input-multi-output multi-band underlay cognitive radio networks', Commun. IET, 2013, 7, (11), pp. 1140-1150.

[9] Abolhasan, M., Lipman, J., Ni, W., Hagelstein, B.: 'Software-defined wireless networking: centralized, distributed, or hybrid', Network, IEEE, 2015, 29, (4), pp. 32-38.

[10] Wang, H., Chen, S., Xu, H., Ai, M., Shi, Y.: 'SoftNet: A software defined decentralized mobile network architecture toward 5G', Network, IEEE, 2015, 29, (2), pp. 16-22. 
[11] Arslan, M., Sundaresan, K., Rangarajan, S.: 'Software-defined networking in cellular radio access networks: potential and challenges', Commun. Mag, IEEE, 2015, 53, (1), pp. 150-156.

[12] Wang, D., Zhang, L., Qi, Y., Quddus, A.U.: 'Localized Mobility Management for SDNIntegrated LTE Backhaul Networks', in Vehicular Technology Conference (VTC Spring), 81st IEEE, 2015, pp. 1-6.

[13] Tuncer, D., Charalambides, M., Clayman, S., Pavlou, G.: 'Adaptive Resource Management and Control in Software Defined Networks', Netw. Serv. Manag. IEEE Trans., 2015, 12, (1), pp. $18-33$.

[14] Yang, C., Chen, Z., Xia, B., Wang, J.: 'When ICN meets C-RAN for HetNets: an SDN approach', Commun. Mag. IEEE, 2015, 53, (11), pp. 118-125.

[15] Musiige, D., Vincent, L., Anton, F., Mioc, D.: LTE RF subsystem power consumption modeling', in Consumer Electronics (GCCE), 2012 IEEE 1st Global Conference on, 2012, pp. 645-649.

[16] Chatzipapas, A., Alouf, S., Mancuso, V.: 'On the minimization of power consumption in base stations using on/off power amplifiers', in Online Conference on Green Communications (GreenCom), 2011 IEEE (2011), pp. 18-23.

[17] Liu, K., He, J., Ding, J., Zhu, Y., Liu, Z.: 'Base station power model and application for energy efficient LTE', in Communication Technology (ICCT), 2013 15th IEEE International Conference on, (2013), pp. 86-92.

[18] Desset, C., Debaillie, B., Giannini, V., et al.: 'Flexible power modeling of LTE base stations', in Wireless Communications and Networking Conference (WCNC), 2012 IEEE (2012), pp. 28582862.

[19] Katranaras, M.I. and E.: 'Energy efficiency analysis of the reference systems, areas of improvements and target breakdown', public Deliv. D2.3 EARTH.

[20] Auer, G., Giannini, V., Desset, C., et al.: 'How much energy is needed to run a wireless network?', Wirel. Commun. IEEE, 2011, 18, (5), pp. 40-49.

[21] Kaup, F., Melnikowitsch, S., Hausheer, D.: 'Measuring and modeling the power consumption of OpenFlow switches', in Network and Service Management (CNSM), 2014 10th International Conference on, (2014), pp. 181-186.

[22] Lee, K.-L., Sedighi, B., Tucker, R.S., Chow, H., Vetter, P.: 'Energy efficiency of optical transceivers in fiber access networks [invited]', Opt. Commun. Networking, IEEE/OSA J., 2012, 4, (9), pp. A59-A68 